\title{
Estimation of Galactic model parameters in high latitudes with the SDSS and SCUSS
}

\author{
Cuihua $\mathrm{Du}^{1}$,Yunpeng $\mathrm{Jia}^{1}$ and Xiyan Peng ${ }^{2}$ \\ ${ }^{1}$ School of Physics, University of Chinese Academy of Sciences, Beijing 100049, China \\ email: ducuihua@ucas.ac.cn \\ ${ }^{2}$ National Astronomical Observatories, Chinese Academy of Sciences, Beijing 100012, China
}

\begin{abstract}
.
Based on the South Galactic Cap U-band Sky Survey (SCUSS) and SDSS observation, we adopted the star-count method to analyze the stellar distribution in different directions of the Galaxy. We find that these model parameters may be variable with observed direction, which cannot simply be attributed to statistical errors.
\end{abstract}

Keywords. Galaxies: structure, Galaxies: fundamental parameters

\section{Estimation of Galactic model parameters}

In this study, we use the South Galactic Cap U-band Sky Survey (SCUSS) and SDSS observation to study the Galactic structure. The adopted model of the Galaxy consists of three components: thin disk, thick disk and halo. From the model fit to the direct measurement of the stellar density distribution, we determine that the range of scale height for the thin disk varies from 180 to 248 pc. The range of scale height for the thick disk is from 400 to $720 \mathrm{pc}$, and the corresponding space number density normalization is $3.5-14.2 \%$ of the thin disk. Our results show that the scale height is variable with the observation direction. The difference between the numerical values of given model parameter estimated for fields with different Galactic longitude can be explained by the influence of disk flare and warp or over-density. It is consistent with merger origin for the thick disk formation. The actual numerical values of Galactic structure parameters are less scientifically important that what they tell us about the Galaxy in general - for example, the origin of the populations. A number of scenarios have been proposed for the origin of the thick disk (Siegel et al. 2002; Ivezić et al. 2008; Jurić M. et al. 2008).

In addition, adopting a de Vaucouleurs $r^{1 / 4}$ law halo, we find that the range of axis ratio is from 0.37 to 0.58 , and the corresponding space number density normalization is $0.145-0.06 \%$ of the thin disk. The axial ratio is dependent upon the observation direction. The reason for the dependence of model parameters on the direction could arise from the asymmetric structure of the halo or over-density and stream accretion from nearby dwarf galaxies. Evidence has been growing for some time that simple description of the Galactic halo are inadequate (Carollo et al. 2008).

\section{References}

Carollo, D., Beers, T. C., Lee, Y. S., 2008, Nature, 451, 216

Ivezić, Ž., et al. 2008, ApJ, 684, 287

Jurić M., Ǐvezić Z., et al. 2008, ApJ, 673, 864

Siegel, M. H., Majewski, S. R., \& Reid, I. N., 2002, ApJ, 578, 151 